\title{
Somatic Sensation of Hand-Object Interactive Movement Is Associated with Activity in the Left Inferior Parietal Cortex
}

\author{
Eiichi Naito ${ }^{1,2,3}$ and H. Henrik Ehrsson ${ }^{1,4}$ \\ ${ }^{1}$ Division of Brain Research, Department of Neuroscience, Karolinska Institute, S-171 77 Stockholm, Sweden, ${ }^{2}$ Graduate School of Human and \\ Environmental Studies, Kyoto University, Sakyo-ku, Kyoto 606-8501, Japan, ${ }^{3}$ ATR Computational Neuroscience Labs, Seika-cho, Soraku-gun, Kyoto 619- \\ 0288, Japan, and ${ }^{4}$ Wellcome Department of Imaging Neuroscience, Institute of Neurology, London WC1N 3BG, United Kingdom
}

Manipulation of objects and tool-use are known to be controlled by a network of frontal motor and parietal areas. Here, we investigate which of these areas are associated with the somatic sensation of hand-object interactive movement using functional magnetic resonance imaging.

To dissociate the sensation of movement from the motor control commands, we used a new kinesthetic illusion. Twelve blindfolded right-handed participants placed the palm of their right or left hand on an object (a ball). Simultaneously, we vibrated the tendon of the wrist extensor muscle. This elicited the illusion that the wrist is flexing and the touched object is also moving along with the hand (hand-object illusion). As controls, we vibrated the skin surface over the nearby bone, which does not elicit any illusions, or we vibrated the tendon when the hand did not touch the object, which only generates the illusory flexion of the hand.

We found that the hand-object illusion specifically activated the left inferior parietal lobule (IPL) (supramarginal gyrus and parietal operculum, including cytoarchitectonic areas ip1 and op1) and area 44. The left IPL was activated both during the hand-object illusions with the right and left hands, and the activity was greater than in the right corresponding parietal region, suggesting a dominant role of the left hemisphere.

We conclude that the left IPL is involved in the somatic perception of hand-object interactive movement and suggest that the underlying mechanism is the somatic integration of internal information about the body and external information about the object.

Key words: kinesthesia; hand-object interaction; inferior parietal lobule; IPL; left hemisphere; normal volunteers; functional magnetic resonance imaging; fMRI

\section{Introduction}

The ability to manipulate objects and use them as tools is a fundamentally important aspect of human hand dexterity. Previous studies on the neuronal basis of object manipulation and tool use have addressed this issue mainly from a motor control perspective (Jeannerod et al., 1995; Binkofski et al., 1999; Ehrsson et al., 2000). However, the tactile and kinesthetic signals from the hand and the fingers are critically important for manual performances (Rothwell et al., 1982; Johansson and Westling, 1984; Johansson et al., 1992). In particular, information about the spatial relationship between the hand and a hand-held object is important when we handle the object. For example, we use a computer mouse without directly watching our hand, because when we hold it, we can sense its location in relation to our hand and body (Graziano and Gross, 1998). Here, we investigate the perceptual aspect of

Received Nov. 10, 2005; revised Feb. 3, 2006; accepted Feb. 20, 2006.

E.N. was supported by the grant-aids for the exchange of foreign research for young scientists from the Ministry of Education, Culture, Sports, Science and Technology (MEXT) of Japan, by a Grant-in-Aid for young scientists from the MEXT Japan, and by the 21st Century COE Program (D-2 to Kyoto University) from the MEXT Japan. H.H.E. was supported by a postdoctoral grant from the Human Frontier Science Program.

Correspondence should be addressed to Dr. Eiichi Naito, Graduate School of Human and Environmental Studies, Kyoto University, Sakyo-ku, Kyoto 606-8501, Japan. E-mail: eiichi.naito@neuro.mbox.media.kyoto-u.ac.jp. DOI:10.1523/JNEUROSCI.4835-05.2006

Copyright $\odot 2006$ Society for Neuroscience $\quad$ 0270-6474/06/263783-08\$15.00/0 hand-object interaction by identifying the neural correlates of the sensation that a touched object is moving with the hand.

To dissociate the sensation of hand-object interactive movement from voluntary motor control processes, we used a kinesthetic illusion that is elicited by vibrating the tendon of a limb (Goodwin et al., 1972). The vibration excites the muscle spindle afferents that signals limb movements (Burke et al., 1976; Roll and Vedel, 1982; Roll et al., 1989). For the present study, we designed a new version of this illusion. Participants touched the surface of a ball with the palm of the right or left hand during which we vibrated the tendon of wrist extensor carpi ulnaris (ECU) muscle (Fig. 1). This elicited the illusion that the wrist is passively flexing and that the touched ball is moving along with the hand (hand-object illusion). This illusion is not associated with any intention to move, sense of effort, or perceived forces between the hand and the object and is thus a purely perceptual illusion (see Results for additional details about the phenomenology of the illusion).

Electrophysiological studies in nonhuman primates have shown that neurons related to object-manipulation are located in the ventral premotor cortex (Murata et al., 1997; Rizzolatti and Luppino, 2001), intraparietal cortex (Murata et al., 2000), superior parietal lobule (Iriki et al., 1996), and inferior parietal lobule (IPL) (Fogassi et al., 2005). Likewise, neuroimaging studies in 


\section{Hand contact}

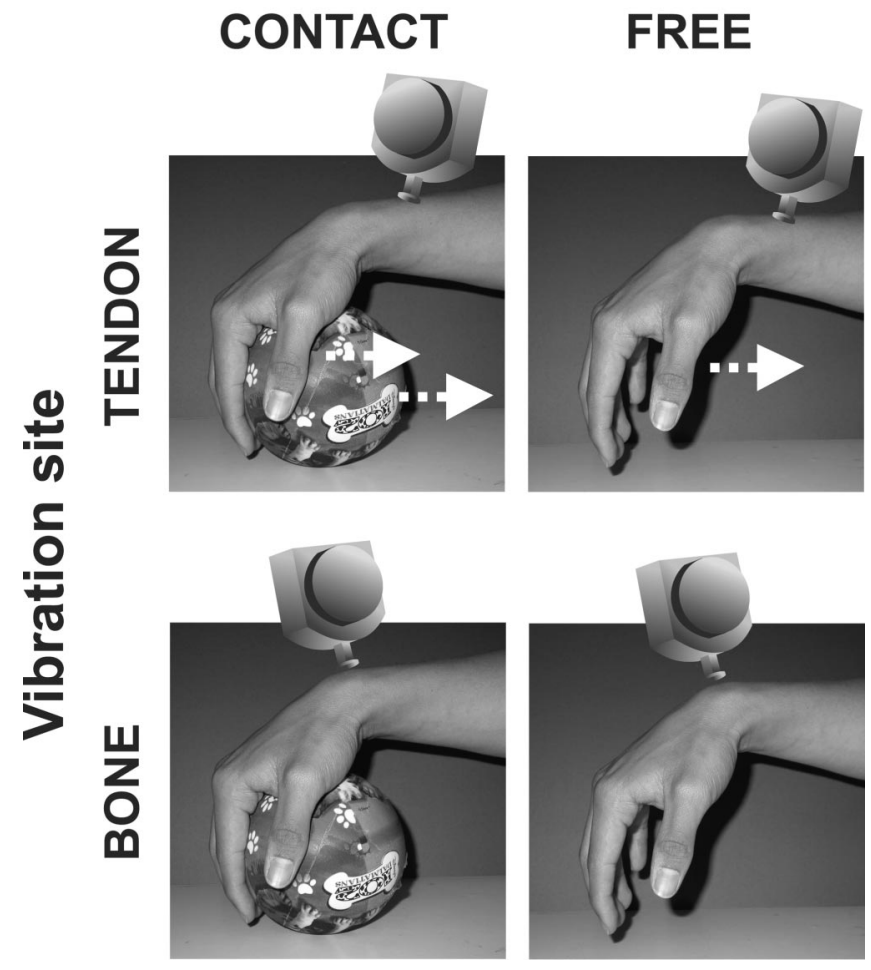

Figure 1. Experimental conditions. To elicit the illusions (top row), we vibrated the tendon of wrist extensor muscles (the ECU; TENDON) of the right or left hand when the palm of the hand rested on the lateral surface of a ball (left column; CONTACT) or when the hand did not touch the ball (right column; FREE). Note that the angle of the wrist was matched in these two situations. To control for the effect of skin vibration, we applied identical stimuli to the skin over the processus styloideus ulnae, which did not elicit any illusions (bottom row; CONTACT-BONE, FREE-BONE).

humans have revealed that inferior-frontal and parietal cortices are activated when we manipulate or explore hand-held objects (Binkofski et al., 1999; Ehrsson et al., 2000, 2001; KuhtzBuschbeck et al., 2001; Stoeckel et al., 2004; Johnson-Frey et al., 2005; Schmitz et al., 2005). Thus, we hypothesized that the somatic sensation of hand-object interactive movements should be associated with activity in some of the frontoparietal areas related to active manipulation of objects, just like the sensation of hand movement involves activation of the primary motor cortex (Naito et al., 1999, 2002b; Naito, 2004). We also predicted a dominance of the left hemisphere, because damages to the left frontoparietal cortices often cause apraxia of tool use (Liepmann, 1905; Schnider et al., 1997; Haaland et al., 2000; Leiguarda and Marsden, 2000; Goldenberg, 2003; Johnson-Frey, 2004). We used functional magnetic resonance imaging (fMRI) to test these hypotheses.

\section{Materials and Methods}

Subjects. Twelve healthy right-handed (Oldfield, 1971) male subjects (21-33 years of age) participated in the study. All subjects gave their informed consent, and the Ethical Committee of the Karolinska Hospital approved the study. The fMRI experiment was performed following the principles and guidelines of the Declaration of Helsinki (1975).

fMRI measurement and tasks. A 1.5 T Signa Horizon Echospeed scanner (General Electrics Medical Systems, Milwaukee, WI) with head-coil provided T1-weighted anatomical images (3D-SPGR) and functional T2*-weighted echoplanar images $(64 \times 64$ matrix, $3.4 \times 3.4 \mathrm{~mm}$; echo time, $60 \mathrm{~ms}$ ). A functional image volume comprised 30 slices of $5 \mathrm{~mm}$ thickness (with $0.4 \mathrm{~mm}$ interslice gap), which ensured that the whole brain was within the field of view.

The subjects were blindfolded, and their ears were plugged. They rested comfortably in a supine position on the bed of the MR scanner. The extended arms were oriented in a prone position parallel to the trunk. The arms were supported proximal to the wrist in a relaxed position. The hands were passively flexed (ranged from 50 to $60^{\circ}$ across subjects) and completely relaxed. During the experimental conditions, the subjects were instructed to relax completely and to be aware of the sensation from the vibrated hand. In some of the experimental conditions, the subjects had their hands positioned so that the palm was in contract with a plastic ball $(r=5 \mathrm{~cm})$ (Fig. 1).

In the experiment, we used a $2 \times 2$ factorial design. One factor was the skin contact with the ball (CONTACT or FREE), and the other was the vibration site (TENDON or BONE). In the CONTACT conditions, the palmar surface of the hand (right or left) was passively in contact with the lateral surface of a ball. In these conditions, the wrist angle was the same as in the FREE conditions, which ensured the equal stretching of wrist muscles, and which in turn means that the vibration stimuli will elicit the same amount of muscle spindle activity. In the FREE conditions, the hands did not touch the ball (Fig. 1). To elicit the illusory palmar flexion of the wrist, we vibrated the tendon of the wrist extensor muscle (the ECU; TENDON). To control for the effect of skin vibration, we applied identical stimuli to the skin over the processus styloideus ulnae, which does not elicit any illusions (BONE). Thus, there were four stimulation conditions (CONTACT-TENDON, CONTACT-BONE, FREE-TENDON, and FREE-BONE), and the participants only experienced the hand-object illusion in the CONTACT-TENDON condition (Fig. 1).

We used a nonmagnetic vibrator, which was driven by constant air pressure provided by an air compressor (BILTEMA Art. 17-635, Linköping, Sweden) (Naito et al., 2002b, 2005). The frequency of the stimulation was $\sim 80 \mathrm{~Hz}$, and the surface of the stylus was $1 \mathrm{~cm}^{2}$. One experimenter in the scanner room manually operated the vibrator by applying it to the skin with a light pressure. To provide the instructions about the conditions and the onset and offset of the vibration to the experimenter, computer-generated visual cues were projected onto the white surface of the scanner (the blindfolded participants could not see this visual information). Two REST conditions in which the subjects relaxed completely and no vibratory stimuli were delivered were also included when the hands touched the ball (CONTACT-REST) or hung freely (FREE-REST).

For each subject, we performed six fMRI sessions: in three of these, the right wrist was vibrated alternating with three sessions in which the left wrist was vibrated (e.g., left session, right session, left session, and so forth). A total of $6 \times 122$ functional image volumes were collected for each subject. In each session, there were six conditions as described above. Each condition lasted for $32 \mathrm{~s}$ (eight functional images; repetition time, $4 \mathrm{~s}$ ) and was repeated twice during each session. The order of conditions was randomized according to a balanced schedule. To remove or replace the ball between the FREE and CONTACT conditions, we included special periods that lasted $16 \mathrm{~s}$ between these conditions. The participants had their hands in contact with the ball for $\sim 4-8 \mathrm{~s}$ before the actual CONTACT condition started. In the analysis, the data from these periods were modeled as conditions of no interest and thereby effectively not used.

Psychophysical testing. In a psychophysical test session conducted a few days before the fMRI, we selected 12 out of 16 participants who experienced vivid illusory palmar flexion when hands were free, and we vibrated the tendon, and who also felt a vivid hand-object illusion when the hands were in contact with the ball. In this test, we vibrated the tendon of the ECU muscle for $30 \mathrm{~s}$. Elecotromyograms (EMGs) were monitored from the skin surface of the vibrated ECU muscle and the flexor muscle [flexor carpi ulnaris (FCU)]. Five trials were performed for each subject. During each trial, the subjects were required to say "start" when they felt that the illusions had started. After each trial, we asked them to verbally describe how they felt during the vibration. To quantify the illusory wrist movements, we asked the subjects to replicate the illusory experiences by actually moving their hands. We measured the maximum wrist angles by 
a protoractor [see methods in the study by Naito et al. (2002a)]. The subjects were also requested to rate the vividness of illusions on an analog scale from 1 to 10 . The vividness was defined as how realistic the illusion was (10 being "absolutely realistic"). In some trials, we also vibrated the tendon of the wrist flexor muscle (FCU) while they touched the ball and asked them about the sensory experiences. Also, in some participants, we vibrated the tendon of the ECU muscle while the palm of the hand was placed on the top of a Coca-Cola can. They reported that the can was shrinking in the direction of the illusory hand flexion. Likewise, when we asked the participants to hold a cylinder and the wrist tendon (flexor or extensor) was vibrated, they reported that the cylinder was moving along with the illusory hand movement. Thus, the hand-object illusions seemed to be highly reproducible regardless of the type of object being touched.

fMRI data analysis. The fMRI data were analyzed with the Statistical Parametric Mapping software (SPM99; http://www.fil.ion.ucl.ac.uk/ spm/; Wellcome Department of Cognitive Neurology, London, UK). The functional images were realigned to correct for head movements (Ashburner and Friston, 1997), coregistered with each subject's anatomical MRI, and transformed (linear and nonlinear transformations) into the reference system of Talairach and Tournoux (1988) (Ashburner and Friston, 1997) using the Montreal Neurological Institute (MNI) reference brain. The functional images were scaled to 100 to correct for global changes in the MR signal, and the images were spatially smoothed with an $8 \mathrm{~mm}$ full-width at half-maximum (FWHM) isotropic Gaussian kernel and smoothed in time by a $4 \mathrm{~s}$ FWHM Gaussian kernel. We fitted a linear regression model (general linear model) to the pooled data from all subjects to increase the sensitivity of the analysis [fixed-effect model, as in Naito et al. (2005) ]. The validity of this approach, in terms of consistency of effects across all subjects in the group, was confirmed by conducting single-subject analyses (see below). We did not use a random-effect model, because our previous experiments indicated that at least 16 subjects are necessary to reliably detect effects related to body image illusions (Ehrsson et al., 2004, 2005). Each condition was modeled with a boxcar function delayed by $4 \mathrm{~s}$ and convoluted with the standard SPM99 hemodynamic response function. Because we knew from the training before the scans that the illusions started after $1.8-2.7 \mathrm{~s}$ (see Results), we omitted the first $4 \mathrm{~s}$ of all conditions by defining these periods as conditions of no interest in the model.

Brain activations related to the hand-object illusions. To identify activity that exclusively related to the hand-object illusions, we examined the interaction between the site of vibration and the skin contact between the hand and the object in the $2 \times 2$ factorial design [(CONTACT-TENDON vs CONTACT-BONE) vs (FREE-TENDON vs FREE-BONE)]. This was done for the right and left hand, separately. We used the contrast of (CONTACT-TENDON vs CONTACT-REST) for the corresponding hand as an inclusive mask ( $p<0.05$, uncorrected) to ensure that only voxels showing activation, as opposed to deactivation, were included. The rationale of this design is that the interaction term reveals activity that specifically reflects the hand-object illusions and that cannot be attributed to the effects of vibrating the muscle tendon or the tactile input from the palm contacting the ball. A voxel-wise threshold of $p<0.001$ uncorrected $(t>3.09)$ was used to generate the cluster images. Because we had a priori anatomical hypothesis that the frontoparietal areas associated with the performance of object manipulation would be active (see Introduction), we restricted the search space and used a small volume correction. We defined our search space as all those voxels that were active in the main effect contrast of all four vibration conditions versus REST ( $p<0.05$, whole brain corrected). This main-effect contrast, which identified a set of bilateral frontoparietal areas, can be used to reduce the search space, because it is orthogonal (i.e., statistically independent) to the interaction contrast. When inspecting the interaction term in the factorial design, we used the threshold of $p<0.05$ corrected based on a test for cluster size.

We also depicted voxels that were active in common during the right and left hand-object illusions (interaction terms). We used the SPM99 conjunction analysis (Price and Friston, 1997; Nichols et al., 2005) ([right (CONTACT-TENDON vs CONTACT-BONE) vs (FREETENDON vs FREE-BONE)] $\cap$ left [(CONTACT-TENDON vs
CONTACT-BONE) vs (FREE-TENDON vs FREE-BONE)]). For this analysis, we used the threshold of $p<0.005$ after the small volume correction described above, which was a sufficiently conservative threshold to ensure that each of the two contrasts in the conjunction analysis corresponded to $p<0.05$ corrected (Nichols et al., 2005).

Single-subject analyses for the interaction. The interaction analyses were based on the functional data pooled across subjects using fixed-effect analyses. The reliability of this model has been criticized, because the results can be biased by a minority of subjects showing very strong effects (Friston et al., 1999). To ensure that the group results were representative for all 12 subjects and to refine the anatomical locations of individual activations in the standard anatomical space $(x, y, z)$, we analyzed the data from individual subjects [as we did in the study by Ehrsson et al. (2003)]. In this purely descriptive analysis, all image-processing steps were identical to those used in the group analysis. The same GLM as in the group analysis was used, with the only difference being that we considered the functional data from each subject separately. We probed for increases in the BOLD signal ( $p<0.05$ uncorrected) in a volume of radius $10 \mathrm{~mm}$ around the voxels, which corresponded to the peaks detected in the group analysis. We report the number of subjects that displayed a BOLD signal increase in the relevant areas. We also summarize peak coordinates in the standard anatomical space $(x, y, z)$ for the left parietal activations observed in the individual subjects and provide the individual $t$ values for these activations (supplemental table, available at www.jneurosci.org as supplemental material).

Testing of dominance of the left hemisphere. When we inspected the activation maps, we found that the left inferior parietal cortex was specifically activated during hand-object illusions no matter whether the right or left hand was tested. No activity was observed in the right inferior parietal cortex (see Results for details). Thus, there appears to be a lateralization to the left hemisphere. However, to statistically test whether this reflects a genuine dominance of the left hemisphere, one has to directly compare the activities in the left regions with those in the right corresponding regions. We did this by flipping (a right-to-left transformation in the $x$-axis) the functional images from all scans of all subjects (the smoothed and normalized images) [see methods in Naito et al. (2005)]. Thus, the right and the left hemispheres were reversed (flipped images). We defined a new general linear model that included both the flipped and the unflipped data. To test whether the BOLD signals in the left regions were significantly greater than those in the right corresponding regions during the hand-object illusions, we defined the contrast [right (CONTACT-TENDON vs CONTACT-BONE) vs right (FREETENDON vs FREE-BONE) vs right-flipped (CONTACT-TENDON vs CONTACT-BONE) vs right-flipped (FREE-TENDON vs FREEBONE)]. The corresponding contrasts were defined to test the left-sided dominance during the left hand-object illusions. Because we were interested in a specific brain region (the left inferior parietal cortex) in this post hoc analysis, we used the threshold of $p<0.05$ corrected after small volume correction in a sphere of radius $10 \mathrm{~mm}$ around the peak voxel identified in this region from the interaction analyses (see Results).

Anatomical definitions. We used cytoarchitecturally defined areas from 10 postmortem brains [cytoarchitectonic area 44 (Amunts et al., 1999), areas IP1 and IP2 in the intraparietal areas (Choi et al., 2006), and areas OP1, OP2, OP3, and OP4 in the parietal operculum (Eickhoff et al., $2006 a, b)]$. A population map was generated for each area (Roland and Zilles, 1998) describing for each voxel how many brains have a representation of one particular cytoarchitectonic area. Each voxel was allocated to the cytoarchitectural area with the highest number of postmortem brains associated with it (Roland and Zilles, 1998). At the "outer" border of a cytoarchitectural area (i.e., at the border of a region where no microstructural data were available), a threshold of 3 of 10 (30\%) brains was applied. This means that voxels with a representation of a given area in $n>3$ brains were assigned to this area (Naito et al., 2002b, 2005). The result of this procedure is a probability map that provides a working definition of the most probable location of each area in the standard anatomical space (Roland et al., 2001). The probability map was finally transformed into the MNI reference brain space (Ashburner and Friston, 1997). This procedure allows us to relate the peak locations of fMRI activation to the cytoarchitectural probability maps. We used these maps 
Table 1. Behavioral results in all TENDON (illusion) conditions

\begin{tabular}{llll}
\hline & Amount (deg) & Vividness & Onset (s) \\
\hline Right CONTACT & $19.4 \pm 6.5$ & $5.9 \pm 1.2$ & $2.3 \pm 1.5$ \\
Left CONTACT & $15.7 \pm 4.2$ & $5.9 \pm 1.0$ & $1.8 \pm 1.0$ \\
& & & \\
Right FREE & $18.4 \pm 4.6$ & $6.0 \pm 1.7$ & $2.7 \pm 2.7$ \\
Left FREE & $18.1 \pm 5.1$ & $6.4 \pm 1.1$ & $1.8 \pm 0.9$ \\
\hline
\end{tabular}

Mean \pm SD across subjects

as "probabilistic indicators" for the anatomical locations of activations in the cytoarchitectural areas. For additional discussion of the technique of combining functional imaging and cytoarchitectural mapping, see studies by Roland and Zilles (1998), Bodegard et al. (2001), Roland et al. (2001), Naito et al. (2002b, 2005), Ehrsson et al. (2003), and Young et al. (2004).

\section{Results}

\section{Psychophysical evaluation}

In the test sessions before the fMRI, we measured the angles of illusory hand movements, determined the onsets of illusions, and recorded the subjective ratings of the vividness of the illusions. When the hand was in contact with the ball (CONTACTTENDON) (Fig. 1), the blindfolded subjects reported the experience that the ball was moving with the hand along the direction of illusory flexion movement. This illusory sensation was highly consistent across trials. The subjects reported no feeling of increasing pressure at the contact surface between the hand and the ball. If the hand was not touching the ball, no hand-object illusion was experienced, even when the distance between the fingers and the object were only $<1 \mathrm{~cm}$. Additionally, we found that the hand-object illusions strictly obeyed the direction of illusory hand movement. When we vibrated the tendon of wrist flexor muscle (creating an illusory extension of the hand), the subjects felt that the ball was "dragged along" the hand. Also, the handobject illusions were often followed by the transient aftereffects (i.e., the sensation that the hand and the ball returned toward the original position within a few seconds). These observations all confirmed that the hand-object illusion is a perceptual illusion that is strictly determined by the pattern of sensory inputs.

Table 1 summarizes the results of the psychophysical tests outside the scanner. The participants experienced a similar amount of illusory wrist movements when the hand was in contact with the ball (CONTACT-TENDON) and when the hand did not touch it (FREE-TENDON). Likewise, the subjects' ratings of the vividness of the illusions were similar between the handobject illusions and the free-hand illusions. In all conditions, the illusions started almost immediately after the start of vibration.

The EMG from the skin surface of the vibrated ECU muscle showed an occasional increase in activity during the tendon vibration (illusions). No reliable differences in EMG activity were observed by visual inspection between the CONTACT conditions and the FREE conditions. From the flexor muscle (the FCU), we found no robust EMG activity during the illusions. These EMG results confirmed those in our previous studies (Naito et al., 2002a,b, 2005).

After each fMRI session, we asked the subjects to verbally describe what they had experienced when we vibrated the tendon. When the hand was free and the tendon was vibrated (FREETENDON), all subjects reported that they experienced illusory hand flexion movements. When the hand was in contact with the ball (CONTACT-TENDON), they reported that they experienced that the touched ball was moving with the hand along its illusory flexion, just as they had felt in the training session. When the skin surface over the nearby bone was vibrated (BONE), the subjects experienced no reliable illusions, as repeatedly confirmed in our previous studies (Naito et al., 2002b, 2005). The experimenter who was standing in the scanner room near the subject observed no overt movements of the subjects' vibrated hands in any conditions.

\section{Brain activation}

Areas specifically active during hand-object illusion

We identified areas that were specifically active during the handobject illusions using the contrast of [(CONTACT-TENDON vs CONTACT-BONE) vs (FREE-TENDON vs FREE-BONE)]. In this contrast, the effects related to the hand contact, the vibration site, and the illusory hand movement were matched, allowing us to reveal activity that specifically reflects the illusion that the touched ball was moving with the hand. The results are summarized in Figure 2 and Table 2.

First, we examined the activations when the left hand was vibrated. Activations were observed in the left frontoparietal cortices ( $p<0.05$, corrected) (Fig. 2, pink sections; Table 2). One cluster was located in the left inferior frontal cortex (cytoarchitectonic area 44) (Fig. 2a,e,f). The other cluster was located in the left IPL, which includes the supramarginal gyrus (SMG) and parietal operculum (PO) (Fig. 2a). In this cluster, we found three peaks. One lay in the most probable location of cytoarchitectonic area ip1 (Fig. 2b). The other two were located in the border zone between areas ip1 and ip2 (Fig. 2c) and area op1 (Fig. 2d). When we tested for the interaction effect in each subject individually (see Materials and Methods), we found activations in the left inferior frontal cortex (area 44) in 10 of 12 subjects and in the left IPL regions in 11 subjects (supplemental table, available at www.jneurosci.org as supplemental material).

Next, we investigated the activity associated with the right hand-object illusion. Such activation was found in the left IPL. Two peaks were located in the border zone between areas ip1 and op1 (Fig. $2 a, c, d, f$, green sections). This activation was located at the similar site where we found the activity associated with the left hand-object illusion (interaction term, see above). From the single-subject analyses, we confirmed that the brain signal increased in the left IPL in 11 subjects (supplemental table, available at www.jneurosci.org as supplemental material). For the inferior frontal cortex (area 44), the signal increased only in five subjects, which may explain why we did not find significant activation in the group analysis.

The left IPL activation was not attributable to different degrees of activation in the hand-object illusions and in the freehand illusions. When we examined the simple contrasts (supplemental information, available at www.jneurosci.org as supplemental material), we found that the hand-object illusion (CONTACT-TENDON vs CONTACT-BONE) activated the left IPL, and that this area was not active during the illusory movement of the hand without the object (FREE-TENDON vs FREEBONE) (supplemental Fig. 1, available at www.jneurosci.org as supplemental material).

Finally, we examined whether the right and left hand-object interactions specifically activated overlapping areas in the left inferior parietal and frontal cortices. The conjunction analysis (see Materials and Methods) showed that both illusory handobject illusions (interaction effects) activated the lateral part of the left IPL in common $[(-63,-39,36) \mathrm{T}=3.04 ; p<0.005$, corrected) (Fig. 2a,c, dark green spots)]. Thus, the perceived hand-object interaction specifically engaged the left IPL regardless of whether the right or left hand was tested. Together, these 


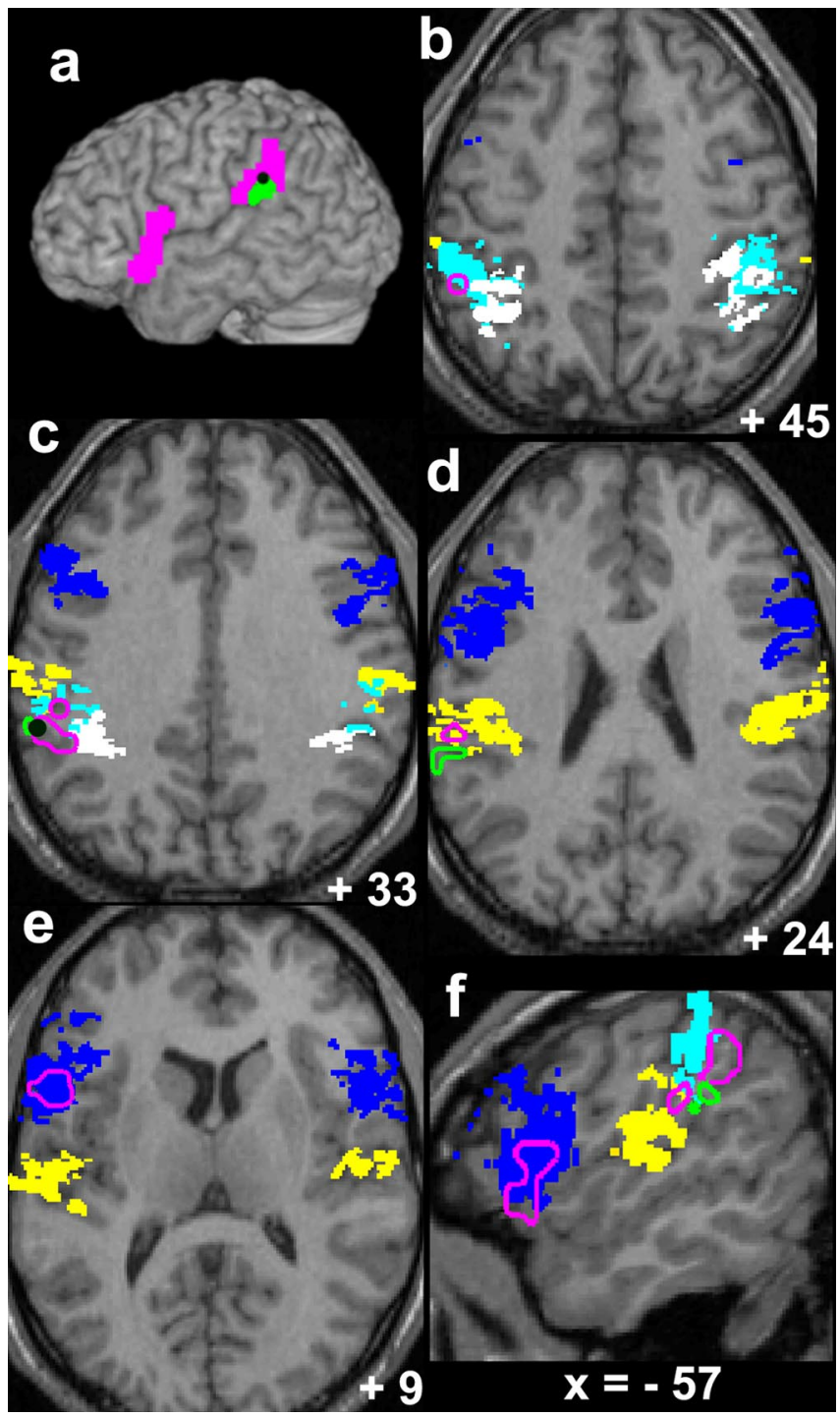

Figure 2. Areas showing activity specifically related to the hand-object illusions [(CONTACTTENDON vs (ONTACT-BONE) vs (FREE-TENDON vs FREE-BONE)] and the cytoarchitectonic areas. The activations are superimposed on the normalized anatomical image of a subject. Pink sections (filled in $\boldsymbol{a}$ and open in $\boldsymbol{b}-\boldsymbol{f}$ ) correspond to the areas activated during the left hand-object illusion; light green sections (filled in $\boldsymbol{a}$ and open in $\boldsymbol{c}, \boldsymbol{d}$, and $\boldsymbol{f}$ ) correspond to those active during the right hand-object illusion. Filled blue sections correspond to the most probable locations of cytoarchitectonic area 44 (light blue, ip1; white, ip2; yellow, op1). The dark green spots in $\boldsymbol{a}$ and c indicate the overlapping areas active during the right and left hand-object interaction.

results strongly suggest that the activity in the left IPL is associated with the somatic sensation that a touched object is moving with the hand regardless of whether the sensation involves the right or left hand.

\section{Dominance of the left hemisphere in the hand-object interaction}

As described above, the left IPL was specifically activated during the illusion of hand-object interaction regardless of whether the right or left hand was used (Fig. $2 a, f$ ). No such activity was observed in the right corresponding region. The flip analysis for the left hand-object interaction showed that the left IPL [peaks $(-45$, $-51,27), \mathrm{T}=4.1 ;(-57,-45,45) \mathrm{T}=3.3 ; p<0.05$, corrected] was significantly more activated than the right corresponding region. Likewise, we found a statistical trend for stronger activity in the left IPL $[(-57,-39,33) \mathrm{T}=2.3 ; p<0.05$, uncorrected $]$

\section{Table 2. Peak coordinates and their T values in hand-object specific activations}

\begin{tabular}{lcccc}
\hline & $x$ & $y$ & $z$ & $T$ \\
\hline $\begin{array}{l}\text { Left hand-object illusion } \\
\text { Left area 44 cluster }\end{array}$ & -57 & 9 & 9 & 4.2 \\
Left IPL cluster & & & & \\
$\quad$ Area ip1 & -57 & -45 & 45 & 4.0 \\
$\quad$ Border area ip1/ip2 & -48 & -48 & 33 & 3.6 \\
$\quad$ Area op1 & -60 & -30 & 24 & 3.7
\end{tabular}

Right hand-object illusion Left IPL cluster

\section{Border area ip1/op1}

Border area ip1/op1

-66
-57

$-42$

27

3.7

$-57$

$-39$

27

than that in the right corresponding region during the right hand-object interaction. Together with the results from the conjunction analysis with the simple contrasts (supplemental information and supplemental Fig. 2, available at www.jneurosci.org as supplemental material), these findings suggest that the somatic sensation that a touched object is moving with the hand predominantly engages the inferior parietal cortices in the left hemisphere.

\section{Discussion}

In the present study, we could associate activity in the left IPL with the somatic sensation that a touched object is moving with the hand. We further demonstrated a dominance of the left hemisphere for this perception. We propose that the left IPL mediates the sensation of hand-object interactive movement by integrating the kinesthetic information of hand movement and haptic information about the external object. These findings provide a neuronal basis of somatic perception of hand-object interaction and also suggest a possible parietal mechanism that links an external object with our own hand when the object or tool becomes "incorporated" into our body image (Maravita and Iriki, 2004).

\section{Hand-object illusion}

To elicit the illusion that the hand and the hand-held object were passively moving, we vibrated the tendon of the wrist muscle while the palm of the hand touched the object. The tendon vibration excites muscle spindle afferents that signal hand movement. These signals are processed by cortical motor areas, cerebellum, and right-sided frontoparietal areas (Naito et al., 1999, 2002a,b, 2005; Naito, 2004) (supplemental Fig. 1, available at www. jneurosci.org as supplemental material). In contrast, the skin contact between the palm and the object may stimulate slow adapting receptors in the skin (Johansson and Vallbo, 1983), which may provide partial information about the stereognostic properties of the object. These tactile signals are processed in the primary somatosensory cortex and the superior parietal lobe (SPL) (Binkofski et al., 1999; Bodegard et al., 2001; Stoeckel et al., 2004). Thus, the hand-object illusion depends on the central integration of kinesthetic and haptic information, which probably takes place in the left IPL (see below).

The hand-object illusion also seems to depend on previous knowledge about the physical and functional properties of the touched object. For example, when people felt illusory flexion of the arm while the hand was placed on the top of a cylinder, they experienced that the cylinder was shrinking along with the illusory arm movement (Naito, 2002). In contrast, when the forearm was fixed on a static wall and the forearm muscles were vibrated while participants were seated on a rotatable chair, most partici- 
pants felt rotation of the entire body (Lackner, 1988). Thus, previous knowledge about the functional properties of the touched objects (i.e., a wall is immobile and a cylinder is shrinkable) may also determine the type of illusory sensation.

\section{Inferior parietal lobule and sensation of hand-object interactive movement}

The present left IPL activation (Fig. 2) does not simply reflect the greater complexity of sensory integration in the hand-object illusion than in the simple wrist flexion illusion. In our previous studies, we investigated other complex illusions, for example, integration between the two hands during illusory bimanual movements (Naito et al., 2002b) and integration between the hands and the waist causing illusory waist shrinkage (Ehrsson et al., 2005). These illusions were not associated with activity in the IPL but activated other areas, namely the primary motor cortex (Naito et al., 2002b) and the junction between the postcentral and intraparietal sulci (Ehrsson et al., 2005), respectively. Thus, the activity in the left IPL reflects the specific type of sensory integration that is needed to elicit the hand-object illusion.

The left IPL activation related to the hand-object illusion was located in the supramarginal gyrus and the parietal operculum. These two parietal areas are strongly associated with the control of object manipulation and tool use in human imaging studies (Binkofski et al., 1999; Ehrsson et al., 2000, 2001; KuhtzBushbeck et al., 2001; Johnson-Frey et al., 2005; Schmitz et al., 2005). Additionally, we observed activity in the left inferior frontal cortex (area 44) during the left hand-object illusion (but not during the right hand-object illusion), which is also an area associated with object manipulation (see above references). The importance of the parietal regions for the perception of hand-object interactive movement might differ from the more motordominant roles of the frontal cortex, as suggested both in humans (Heilman et al., 1982; Binkofski et al., 2001) and in nonhuman primates (Murata et al., 1997, 2000). Nevertheless, the sensation of hand-object interactive movements seems to engage some of the areas that are activated during active object manipulation. We have shown previously that the primary motor cortex has a role in the somatic perception of limb movements, in addition to its executive role of producing limb movements (Naito et al., 2002b; Naito, 2004). Thus, the present finding supports the general hypothesis that actions and their perceptual contents may share some of the same brain areas (Naito et al., 1999, 2002b; Hommel et al., 2001; Naito and Sadato, 2003; Berti et al., 2005).

Recently, it has been demonstrated that human inferior parietal regions can be cytoarchitecturally subdivided into several distinct regions (areas IP1-2, OP1-4) (Choi et al., 2006; Eickhoff et al., 2006a,b). Area IP1 is caudally located to area 2, lines the rostral part of the intraparietal sulcus, and extends into the SMG. Area IP2 is caudally and somewhat medially located to area IP1. Area OP1 is located laterally in the PO and also extends dorsally into the SMG (Young et al., 2004; Naito et al., 2005). These areas are considered to be high-order somatosensory areas in humans (Young et al., 2004; Naito et al., 2005).

The precise homology between the cytoarchitectural areas in human and monkey's inferior parietal lobules is still not known (Eidelberg and Galaburda, 1984). In the present study, the activations associated with the hand-object illusions were located in the lateral-most parts of the left inferior parietal regions, including cytoarchitectonic areas ip1 and op1 (Fig. 2), regardless of which hand was used. The lateral and posterior part of the monkey's inferior parietal lobule (area 7b/PF) contains neurons that predominantly respond to somaesthetic inputs and are involved in cutaneous-somatomotor integration (Hyvärinen, 1982). Likewise, neurons in the parietal opercular region (SII) also respond to various types of somatosensory stimuli (Robinson and Burton, 1980). Importantly, the monkey's PF and SII project to area F5, which perhaps corresponds to the human ventral premotor cortex or area 44 (Godschalk et al., 1984; Matelli et al., 1986; Rizzolatti and Luppino, 2001). Thus, our view that the kinesthetic information of hand movement and haptic information about the external object are integrated in the parietal regions to elicit the sensation of hand-object interactive movements seems to be consistent with the physiological and anatomical properties of these areas.

Finally, in the present study, we did not observe activity in the SPL. We know from previous experiments that the SPL is important for haptic perception of objects (Binkofski et al., 2001; Stoeckel et al., 2004) and that this region is recruited during the active exploration of objects (Binkofski et al., 1999). In the present study, the haptic inputs were matched in the interaction contrast, which probably explains the lack of SPL activity. In contrast, the IPL seems to be important for representing the spatial relationship between the hand and the hand-held object (i.e., position and movement of the object relative to the hand).

\section{Dominance of the left hemisphere for the sensation of hand-object interaction}

We found that the left IPL activation was related to the handobject illusion regardless of which hand was used (Fig. 2), and that no such activation was observed in the right corresponding parietal regions (supplemental Fig. 2, available at www.jneurosci.org as supplemental material). This reflects a genuine lateralization to the left hemisphere as shown in the present flip analyses (see Results). Thus, our data provide direct physiological evidence for a dominant role of the left hemisphere in the somatic perception of hand-object interaction.

This finding may provide a physiological basis for the neurological observations that lesions in the left hemisphere that include inferior parietal cortices often cause ideomotor apraxia (Liepmann, 1905; Schnider et al., 1997; Haaland et al., 2000; Leiguarda and Marsden, 2000; Goldenberg, 2003; Johnson-Frey, 2004). The apraxia after left parietal damage is often present when using either hand (Alexander et al., 1992; Haaland and Harrington, 1996; Leiguarda and Marsden, 2000; Halsband et al., 2001). Additionally, our results seem to be consistent with a meta-analysis of parietal activation studies (Nickel and Seitz, 2005), which suggests that the left IPL is involved in the handobject interaction, and with Heilman et al. (1982), who, based on an analysis of human lesion data, suggested that the inferior parietal lobule (supramarginal gyrus) contains visuokinesthetic representations (i.e., perceptual representations related to the motor performances with objects and tools). The clinical observation that apraxia often coexists with aphasia (De Renzi et al., 1980; Haaland and Flaherty, 1984; Kertesz et al., 1984; Papagno et al., 1993) may reflect that both hand-object interaction and language (Binder et al., 1997; Vikingstad et al., 2000) are lateralized to the left hemisphere.

The lateralization to the left IPL for the hand-object interaction is strikingly different from the lateralization to the right corresponding regions for the perception of simple limb movements (Naito et al., 2005). In the present study, we reproduced the finding that right inferior parietal and inferior frontal cortices are active when the participants experience illusory movements of either hand without touching the object (supplemental Fig. $2 c, d$, available at www.jneurosci.org as supplemental material). 
Thus, it seems that the right parietal regions are specialized for neuronal processing of information from one's own body (Berti et al., 2005; Naito et al., 2005), whereas the left parietal regions are specialized for the neuronal association between the internal (body) and the external (objects) information (Haaland and Flaherty, 1984).

\section{References}

Alexander MP, Baker E, Naeser MA, Kaplan E, Palumbo C (1992) Neuropsychological and neuroanatomical dimensions of ideomotor apraxia. Brain 115:87-107.

Amunts K, Schleicher A, Burgel U, Mohlberg H, Uylings HBM, Zilles K (1999) Broca's region revisited: cytoarchitecture and intersubject variability. J Comp Neurol 412:319-341.

Ashburner J, Friston KJ (1997) Spatial transformation of images. In: Human brain function (Frackowiak RSJ, Friston KJ, Frith CD, Dolan RJ, Mazziotta JC, ed), pp 43-58. San Diego: Academic.

Berti A, Bottini G, Gandola M, Pia L, Smania N, Stracciari A, Castiglioni I, Vallar G, Paulesu E (2005) Shared cortical anatomy for motor awareness and motor control. Science 309:488-491.

Binder JR, Frost JA, Hammeke TA, Cox RW, Rao SM, Prieto T (1997) Human brain language areas identified by functional magnetic resonance imaging. J Neurosci 17:353-362.

Binkofski F, Buccino G, Posse S, Seitz RJ, Rizzolatti G, Freund HJ (1999) A fronto-parietal circuit for object manipulation in man: evidence from an fMRI-study. Eur J Neurosci 11:3276-3286.

Binkofski F, Kunesch E, Classen J, Seitz RJ, Freund HJ (2001) Tactile apraxia: unimodal apractic disorder of tactile object exploration associated with parietal lobe lesions. Brain 124:132-144.

Bodegard A, Geyer S, Grefkes C, Zilles K, Roland PE (2001) Hierarchical processing of tactile shape in the human brain. Neuron 31:317-328.

Burke D, Hagbarth KE, Lofstedt L, Wallin BG (1976) The responses of human muscle spindle endings to vibration of non-contracting muscles. J Physiol (Lond) 261:673-693.

Choi HJ, Zilles K, Mohlberg H, Schleicher A, Fink GR, Armstrong E, Amunts K (2006) Cytoarchitectonic identification and probabilistic mapping of two distinct areas within the anterior ventral bank of the human intraparietal sulcus. J Comp Neurol 495:53-69.

De Renzi E, Motti F, Nichelli P (1980) Imitating gestures. A quantitative approach to ideomotor apraxia. Arch Neurol 37:6-10.

Ehrsson HH, Fagergren E, Jonsson T, Westling G, Johansson RS, Forssberg H (2000) Cortical activity in precision versus power-grip tasks: an fMRI study. J Neurophysiol 83:528-536.

Ehrsson HH, Fagergren E, Forssberg H (2001) Differential fronto-parietal activation depending on force used in a precision grip task: an fMRI study. J Neurophysiol 85:2613-2623.

Ehrsson HH, Geyer S, Naito E (2003) Imagery of voluntary movement of fingers, toes, and tongue activates corresponding body-part-specific motor representations. J Neurophysiol 90:3304-3316.

Ehrsson HH, Spence C, Passingham RE (2004) That's my hand! Activity in the premotor cortex reflects feeling of ownership of a limb. Science 305:875-877.

Ehrsson HH, Kito T, Sadato N, Passingham RE, Naito E (2005) Neural substrate of body size: illusory feeling of shrinking of the waist. PLoS Biol 3:e412.

Eickhoff SB, Schleicher A, Zilles K, Amunts K (2006a) The human parietal operculum. I. Cytoarchitectonic mapping of subdivisions. Cereb Cortex 16:254-267.

Eickhoff SB, Amunts K, Mohlberg H, Zilles K (2006b) The human parietal operculum. II. Stereotaxic maps and correlation with functional imaging results. Cereb Cortex 16:268-279.

Eidelberg D, Galaburda AM (1984) Inferior parietal lobule. Divergent architectonic asymmetries in the human brain. Arch Neurol 41:843-852.

Fogassi L, Ferrari PF, Gesierich B, Rozzi S, Chersi F, Rizzolatti G (2005) Parietal lobe: from action organization to intention understanding. Science 308:662-667.

Friston KJ, Holmes AP, Worsley KJ (1999) How many subjects constitute a study? NeuroImage 10:1-5.

Godschalk M, Lemon RN, Kuypers HGJM, Ronday HK (1984) Cortical afferents and efferents of monkey postarcuate area: an anatomical and electrophysiological study. Exp Brain Res 56:410-424.
Goldenberg G (2003) Apraxia and beyond: life and work of Hugo Liepmann. Cortex 39:509-524.

Goodwin GM, McCloskey DI, Matthews PBC (1972) Proprioceptive illusions induced by muscle vibration: contribution by muscle spindles to perception? Science 175:1382-1384.

Graziano MS, Gross CG (1998) Spatial maps for the control of movement. Curr Opin Neurobiol 8:195-201.

Haaland KY, Flaherty D (1984) The different types of limb apraxia errors made by patients with left vs. right hemisphere damage. Brain Cogn 3:370-384.

Haaland KY, Harrington DL (1996) Hemispheric asymmetry of movement. Curr Opin Neurobiol 6:796-800.

Haaland KY, Harrington DL, Knight RT (2000) Neural representations of skilled movement. Brain 123:2306-2313.

Halsband U, Schmitt J, Weyers M, Binkofski F, Grutzner G, Freund HJ (2001) Recognition and imitation of pantomimed motor acts after unilateral parietal and premotor lesions: a perspective on apraxia. Neuropsychologia 39:200-216.

Heilman KM, Rothi LJ, Valenstein E (1982) Two forms of ideomotor apraxia. Neurology 32:342-346.

Hommel B, Musseler J, Aschersleben G, Prinz W (2001) The theory of event coding (TEC): a framework for perception and action planning. Behav Brain Sci 24:849-937.

Hyvärinen J (1982) Studies of brain function. The parietal cortex of monkey and man, pp 48-139. Berlin: Springer.

Iriki A, Tanaka M, Iwamura Y (1996) Coding of modified body schema during tool use by macaque postcentral neurones. NeuroReport 7:2325-2330.

Jeannerod M, Arbib MA, Rizzolatti G, Sakata H (1995) Grasping objects: the cortical mechanisms of visuomotor transformation. Trends Neurosci 18:314-320.

Johansson RS, Vallbo AB (1983) Tactile sensory coding in the glabrous skin of the human hand. Trends Neurosci 6:27-32.

Johansson RS, Westling G (1984) Roles of glabrous skin receptors and sensorimotor memory in automatic control of precision grip when lifting rougher or more slippery objects. Exp Brain Res 56:550-564.

Johansson RS, Hger C, Backstrom L (1992) Somatosensory control of precision grip during unpredictable pulling loads. III. Impairments during digital anesthesia. Exp Brain Res 89:204-213.

Johnson-Frey SH (2004) The neural bases of complex tool use in humans. Trends Cogn Sci 8:71-78.

Johnson-Frey SH, Newman-Norlund R, Grafton ST (2005) A distributed left hemisphere network active during planning of everyday tool use skills. Cereb Cortex 15:681-695.

Kertesz A, Ferro JM, Shewan CM (1984) Apraxia and aphasia: the functional-anatomical basis for their dissociation. Neurology 34:40-47.

Kuhtz-Buschbeck JP, Ehrsson HH, Forssberg H (2001) Human brain activity in the control of fine static precision grip forces: an fMRI study. Eur J Neurosci 14:382-390.

Lackner JR (1988) Some proprioceptive influences on the perceptual representation of body shape and orientation. Brain 111:281-297.

Leiguarda RC, Marsden CD (2000) Limb apraxias: higher-order disorders of sensorimotor integration. Brain 123:860-879.

Liepmann H (1905) Der weitere Krankheitsverlauf bei dem einseitig Apraktischen und der Gehirnbefund auf Grund von Serienschnitten. Monatschr Psychiat Neurol 17:289-311, 19:217-243.

Maravita A, Iriki A (2004) Tools for the body (schema). Trends Cogn Sci 8:79-86.

Matelli M, Camarda R, Glickstein M, Rizzolatti G (1986) Afferent and efferent projections of the inferior area 6 in the macaque monkey. J Comp Neurol 251:281-298.

Murata A, Fadiga L, Fogassi L, Gallese V, Raos V, Rizzolatti G (1997) Object representation in the ventral premotor cortex (area F5) of the monkey. J Neurophysiol 78:2226-2230.

Murata A, Gallese V, Luppino G, Kaseda M, Sakata H (2000) Selectivity for the shape, size, and orientation of objects for grasping in neurons of monkey parietal area AIP. J Neurophysiol 83:2580-2601.

Naito E (2002) Perceptual distortion of intrapersonal and near-personal space sensed by proprioception. Percep Mot Skills 94:499-505.

Naito E (2004) Sensing limb movements in the motor cortex: how humans sense limb movement. Neuroscientist 9:73-82. 
Naito E, Sadato N (2003) Internal simulation of expected sensory experiences before movements get started. Rev Neurosci 14:387-399.

Naito E, Ehrsson HH, Geyer S, Zilles K, Roland PE (1999) Illusory arm movements activate cortical motor areas: a PET study. J Neurosci 19:6134-6144.

Naito E, Kochiyama T, Kitada R, Nakamura S, Matsumura M, Yonekura Y, Sadato N (2002a) Internally simulated movement sensations during motor imagery activate the cortical motor areas and the cerebellum. J Neurosci 22:3683-3691.

Naito E, Roland PE, Ehrsson HH (2002b) I feel my hand moving: a new role of the primary motor cortex in somatic perception of limb movement. Neuron 36:979-988.

Naito E, Roland PE, Grefkes C, Choi HJ, Eickhoff S, Geyer S, Zilles K, Ehrsson $\mathrm{HH}$ (2005) Dominance of the right hemisphere and role of area 2 in human kinesthesia. J Neurophysiol 93:1020-1034.

Nichols T, Brett M, Andersson J, Wager T, Poline JB (2005) Valid conjunction inference with the minimum statistic. NeuroImage 25:653-660.

Nickel J, Seitz RJ (2005) Functional clusters in the human parietal cortex as revealed by an observer-independent meta-analysis of functional activation studies. Anat Embryol 210:463-472.

Oldfield RC (1971) The assessment and analysis of handedness: the Edinburgh inventory. Neuropsychologia 9:97-113.

Papagno C, Della Sala S, Basso A (1993) Ideomotor apraxia without aphasia and aphasia without apraxia: the anatomical support for a double dissociation. J Neurol Neurosurg Psychiatry 56:286-289.

Price CJ, Friston KJ (1997) Cognitive conjunction: a new approach to brain activation experiments. NeuroImage 5:261-270.

Rizzolatti G, Luppino G (2001) The cortical motor system. Neuron 31:889-901.

Robinson CJ, Burton H (1980) Somatic submodality distribution within the second somatosensory (SII), 7b, retroinsular, postauditory, and granular insular cortical areas of M. fascicularis. J Comp Neurol 192:93-108.
Roland PE, Zilles K (1998) Structural divisions and functional fields in the human cerebral cortex. Brain Res Brain Res Rev 26:87-105.

Roland PE, Svensson G, Lindeberg T, Risch T, Baumann P, Dehmel A, Frederiksson J, Halldorson H, Forsberg L, Young J, Zilles K (2001) A database generator for human brain imaging. Trends Neurosci 24:562-564.

Roll JP, Vedel JP (1982) Kinaesthetic role of muscle afferent in man, studied by tendon vibration and microneurography. Exp Brain Res 47:177-190.

Roll JP, Vedel JP, Ribot E (1989) Alteration of proprioceptive messages induced by tendon vibration in man: a microneurographic study. Exp Brain Res 76:213-222.

Rothwell JC, Traub MM, Day BL, Obeso JA, Thomas PK, Marsden CD (1982) Manual motor performance in a deafferented man. Brain 105:515-542.

Schmitz C, Jenmalm P, Ehrsson HH, Forssberg H (2005) Brain activity during predictable and unpredictable weight changes when lifting objects. J Neurophysiol 93:1498-1509.

Schnider A, Hanlon RE, Alexander DN, Benson DF (1997) Ideomotor apraxia: behavioral dimensions and neuroanatomical basis. Brain Lang 58:125-136.

Stoeckel MC, Weder B, Binkofski F, Choi HJ, Amunts K, Pieperhoff P, Shah NJ, Seitz RJ (2004) Left and right superior parietal lobule in tactile object discrimination. Eur J Neurosci 19:1067-1072.

Talairach J, Tournoux P (1988) A co-planar stereotaxic atlas of a human brain. New York: Thieme.

Vikingstad EM, George KP, Johnson AF, Cao Y (2000) Cortical language lateralization in right handed normal subjects using functional magnetic resonance imaging. J Neurol Sci 175:17-27.

Young JP, Herath P, Eickhoff S, Choi HJ, Grefkes C, Zilles K, Roland PE (2004) Somatotopy and attentional modulation of the human parietal and opercular regions. J Neurosci 24:5391-5399. 